\title{
Excitation gap of a graphene channel with superconducting boundaries
}

\author{
M. Titov, ${ }^{1}$ A. Ossipov, ${ }^{2}$ and C. W. J. Beenakker ${ }^{2}$ \\ ${ }^{1}$ Department of Physics, Konstanz, University, D-78457 Konstanz, Germany \\ ${ }^{2}$ Instituut-Lorentz, Universiteit Leiden, P.O. Box 9506, 2300 RA Leiden, The Netherlands
}

(Received 25 September 2006; published 11 January 2007)

\begin{abstract}
We calculate the density of states of electron-hole excitations in a superconductor-normal-metalsuperconductor (SNS) junction in graphene, in the long-junction regime that the superconducting gap $\Delta_{0}$ is much larger than the Thouless energy $E_{T}=\hbar v / d$ (with $v$ the carrier velocity in graphene and $d$ the separation of the NS boundaries). If the normal region is undoped, the excitation spectrum consists of neutral modes that propagate along the boundaries - transporting energy but no charge. These "Andreev modes" are a coherent superposition of electron states from the conduction band and hole states from the valence band, coupled by specular Andreev reflection at the superconductor. The lowest Andreev mode has an excitation gap of $E_{0}$ $=\frac{1}{2}(\pi-|\phi|) E_{T}$, with $\phi \in(-\pi, \pi)$ the superconducting phase difference. At high doping (Fermi energy $\left.\mu \gg E_{T}\right)$ the excitation gap vanishes $\propto E_{0}\left(E_{T} / \mu\right)^{2}$, and the usual gapless density of states of Andreev levels is recovered. We use our results to calculate the $\phi$ dependence of the thermal conductance of the graphene channel.
\end{abstract}

DOI: 10.1103/PhysRevB.75.045417

PACS number(s): 74.45.+c, 73.20.At, 73.23.Ad, 74.78.Na

\section{INTRODUCTION}

The two-dimensional layer of carbon atoms known as graphene is a gapless semiconductor. A gap between conduction and valence bands opens up if the layer is confined to a narrow channel. ${ }^{1}$ For a channel of width $d$ the band gap $2 E_{0}$ is set by the (ballistic) Thouless energy $E_{T}=\hbar v / d$, with $v$ the (energy independent) velocity of electron and hole excitations in graphene. The size of the gap depends on the crystallography of the channel edges. In particular, for edges in the armchair configuration one has ${ }^{2,3}$

$$
E_{0}=\alpha E_{T},
$$

with $\alpha=0$ if the channel is a multiple of three unit cells across or $\alpha=\pi / 3$ otherwise.

The interface with a superconductor provides an altogether different way to confine the carriers. At energies below the superconducting gap $\Delta_{0}$, the electron and hole excitations in a superconductor-normal-metal-superconductor (SNS) junction are confined to the normal region. In usual metals this confinement leads to bound states known as Andreev levels. ${ }^{4,5}$ They consist of counterpropagating electrons and holes converted into each other by Andreev retroreflection at the NS boundaries [see Fig. 1(a)]. Andreev levels carry an electrical current (a supercurrent) across the NS interfaces, but they are "quasilocalized" along the interfaces. More precisely, the group velocity of the Andreev levels along the NS interface is much smaller than the Fermi velocity, and weak disorder fully localizes them. ${ }^{6}$

As pointed out in Ref. 7, Andreev reflection in undoped graphene is specular reflection instead of retro-reflection [see Fig. 1(b)]. The consequences were investigated in that paper and in Ref. 8 for a single NS interface, and in Refs. 9 and 10 for a short SNS junction. Here we investigate the consequences for a long SNS junction.

We find that the transition from retro-reflection to specular reflection is accompanied by a transition from quasilocalized Andreev levels to propagating modes ("Andreev modes"), provided that $E_{T} \ll \Delta_{0}$. This is the long-junction re- gime. (The states remain localized in the opposite shortjunction regime $E_{T} \gg \Delta_{0}$, considered in Ref. 9.) The transition (governed by the ratio $\mu / E_{T}$ of the Fermi energy $\mu$ in $N$ and the Thouless energy) has a drastic effect on the density of states. While the excitation spectrum is gapless for $\mu$ $\gg E_{T}$, a gap opens up for $\mu \lesssim E_{T}$. The excitation gap

$$
E_{0}=\frac{1}{2}(\pi-|\phi|) E_{T}
$$

has the same form as the band gap (1) for confinement by armchair edges - with the phase difference $\phi \in(-\pi, \pi)$ of the two superconductors taking over from the crystallographic phase $\alpha$.

The Andreev modes have the same dispersion relation as the "armchair modes" for confinement by armchair edges, and they are also constructed out of states taken from two different valleys in the Brillouin zone. However, while the armchair modes contain either electron states from the con-

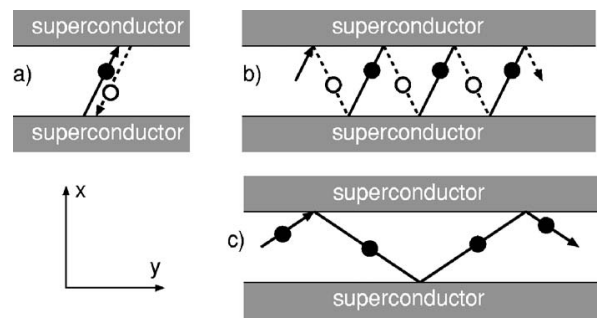

FIG. 1. Three types of states in an SNS channel in graphene. The solid and dashed lines show the classical trajectories of an electron (filled circle) and a hole (open circle), converted into each other upon Andreev reflection at the superconductor. The transition from a localized Andreev level (a) to a propagating Andreev mode (b) occurs when the excitation energy $\varepsilon$ becomes larger than the Fermi energy $\mu$ in the normal region. These two types of states are both charge neutral. Purely electronic states (c) exist near grazing incidence. While the states of type (a) and (b) are sensitive to the phase difference $\phi$ of the two superconductors, the states of type (c) are not. 
duction band or hole states from the valence band, the Andreev modes are a superposition of conduction and valence band states. As a consequence, the Andreev modes transport energy but no charge along the NS interface-so they will play a role in thermal conduction along the interface but not in electrical conduction.

The outline of this paper is as follows. The modes propagating along the channel are characterized by their dispersion relation in Sec. II. Both exact numerical and approximate (but highly accurate) analytical results are given. From the dispersion relation we determine the excitation gap in Sec. III and the density of states in Sec. IV, contrasting in particular the low- and high-doping regimes. We derive the result (2) for the excitation gap in the low-doping regime and show numerically that the gap closes $\propto E_{0}\left(E_{T} / \mu\right)^{2}$ with increasing doping. One way to measure the gap is by tunneling spectroscopy. Another way, which we analyze in some detail in Sec. V, is by means of the thermal conductance of the channel (for heat flow parallel to the NS boundaries). We conclude in Sec. VI.

\section{DISPERSION RELATION}

\section{A. Quantization condition}

To calculate the dispersion relation of the Andreev modes we solve the Dirac-Bogoliubov-De Gennes (DBdG) equation $^{7}$ for the pair potential

$$
\Delta(\boldsymbol{r})=\left\{\begin{array}{cc}
\Delta_{0} \exp (i \phi / 2) & \text { if } x<-d / 2 \\
0 & \text { if }-d / 2<x<d / 2 \\
\Delta_{0} \exp (-i \phi / 2) & \text { if } x>d / 2
\end{array}\right.
$$

We seek plane-wave solutions $\Psi(x, y)=\psi(x) e^{i q y}$, with $q$ the component of the wave vector parallel to the NS interfaces at $x= \pm d / 2$. The excitation energy $\varepsilon>0$ of the mode is measured relative to the Fermi energy $\mu$ in the normal region $|x|<d / 2$. (The superconducting regions are assumed to be heavily doped, with Fermi energy $\mu^{\prime} \gg \mu$.)

The dispersion relation follows from the quantization condition derived from the $\mathrm{DBdG}$ equation in Ref. 9,

$$
\begin{aligned}
\cos \phi= & \left(\cos \theta_{+} \cos \theta_{-}+\frac{\sin \theta_{+} \sin \theta_{-}}{\cos \alpha_{+} \cos \alpha_{-}}\right) \cos 2 \beta \\
& +\left(\frac{\sin \theta_{+} \cos \theta_{-}}{\cos \alpha_{+}}-\frac{\cos \theta_{+} \sin \theta_{-}}{\cos \alpha_{-}}\right) \sin 2 \beta \\
& -\sin \theta_{+} \sin \theta_{-} \tan \alpha_{+} \tan \alpha_{-}
\end{aligned}
$$

The three angles $\alpha_{ \pm}, \theta_{ \pm}, \beta$ are functions of $\varepsilon$ and $q$,

$$
\begin{gathered}
\alpha_{ \pm}=\arcsin \left(\frac{q}{\mu \pm \varepsilon}\right), \quad \theta_{ \pm}=\frac{\mu \pm \varepsilon}{E_{T}} \cos \alpha_{ \pm}, \\
\beta=\arccos \left(\varepsilon / \Delta_{0}\right) .
\end{gathered}
$$

The quantization condition is invariant under $\mu \rightarrow-\mu$, so without loss of generality we may take $\mu>0$.

While Ref. 9 dealt with the short-junction regime $E_{T}$ $\gg \Delta_{0}$, here we are concerned with the long-junction regime $E_{T} \ll \Delta_{0}$. (Since the Thouless energy $E_{T} \equiv \hbar v / d$, the latter criterion is equivalent to the requirement that the separation $d$ of the NS interfaces is large compared to the superconducting coherence length $\xi \equiv \hbar v / \Delta_{0}$.) We furthermore restrict ourselves to low-lying excitations, $\varepsilon \ll \Delta_{0}$. The relative magnitude of $\varepsilon$ and $E_{T}$ is arbitrary. For ease of notation we will use units such that $\hbar v \equiv 1$ in the intermediate calculations, restoring the units in the final results.

For low-lying excitations $\varepsilon \ll \Delta_{0}$ the quantization condition (4) simplifies to

$$
\cos \phi+\cos \theta_{+} \cos \theta_{-}+r \sin \theta_{+} \sin \theta_{-}=0,
$$

where we have abbreviated

$$
r=\frac{1+\sin \alpha_{+} \sin \alpha_{-}}{\cos \alpha_{+} \cos \alpha_{-}}
$$

The solutions to this equation can be represented in the form $\varepsilon=\varepsilon_{n}^{ \pm}(q)$, where $n=0,1,2, \ldots$ is the mode index due to the quantization of the motion in the $x$ direction and the superscript \pm accounts for the different $\phi$ dependence of the modes.

\section{B. Exact solution}

The quantization condition (7) can be solved numerically. Results are shown in Figs. 2, 3, and 4. Only positive $q$ is plotted, because $\varepsilon_{n}^{ \pm}(-q)=\varepsilon_{n}^{ \pm}(q)$.

The dispersion relation has three distinctly different branches, indicated in Fig. 4, corresponding to the three types of trajectories of Fig. 1.

(i) The branch with $\hbar v|q|<\mu-\varepsilon$ (red) describes intra band electron-hole states, corresponding to the Andreev modes of Fig. 1(a). The dispersion relation for these modes has small oscillations as a function of $q$ on the scale $1 / d$, around a smooth concave curve (see Fig. 3). Similar oscillations in the dispersion relation of Andreev modes in mesososcopic samples were discussed recently in Ref. 11.

(ii) The branch with $\hbar v|q|<\varepsilon-\mu$ (blue) describes inter band electron-hole states, corresponding to the Andreev modes of Fig. 1(b). The dispersion relation is convex without oscillations.

(iii) The branch with $\hbar v|q|>|\varepsilon-\mu|$ (green) corresponds to the purely electronic states ${ }^{12}$ of Fig. 1(c). The hole component of the wave function cannot propagate along the channel because the reflection angle $\alpha_{-}$of the hole is imaginary on this branch. The dispersion relation on branch (iii) does not have oscillations, and is joined to branch (i) or (ii) by a cusp singularity.

After these exact results we continue with an approximate, but highly accurate, analytical solution of the quantization condition. We consider separately the electron-hole modes with $\hbar v|q|<|\varepsilon-\mu|$ and the electron modes with $\hbar v|q|>|\varepsilon-\mu|$. 


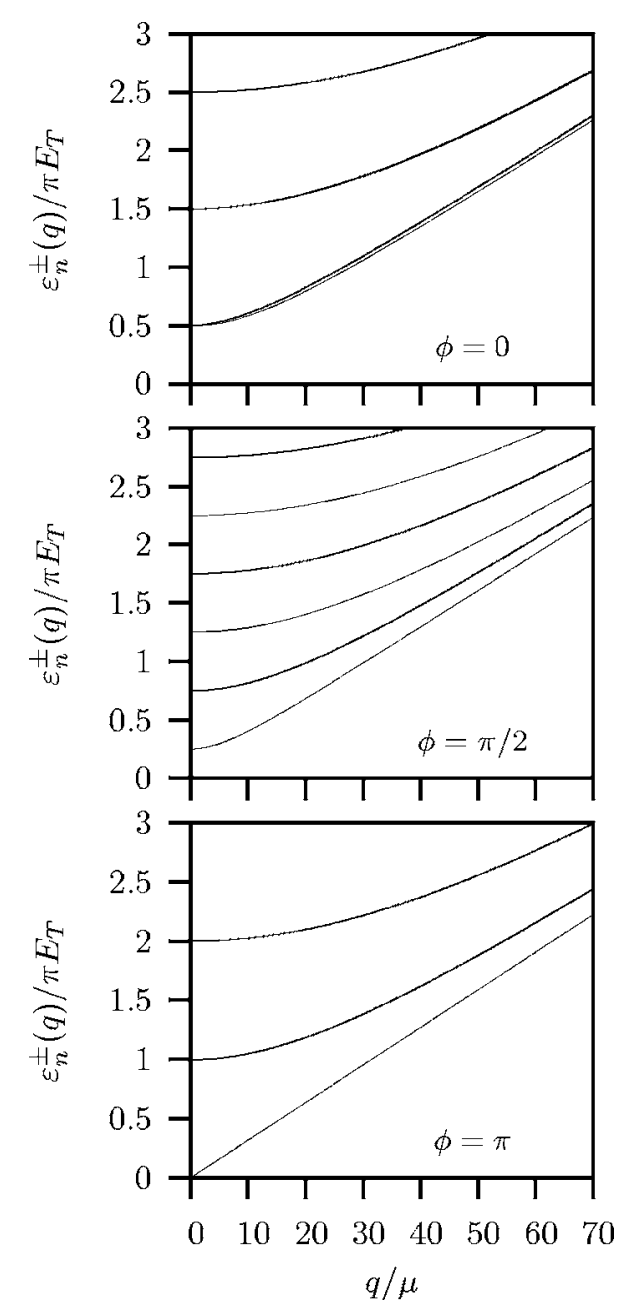

FIG. 2. Dispersion relation of the SNS junction, calculated numerically from Eq. (7) for three values of the superconducting phase difference $\phi$ at $\mu / E_{T}=0.1$. The lowest modes $\varepsilon_{n}^{ \pm}(q)$ with $n$ $=0,1,2$ are nearly degenerate for $\phi=0$ and nondegenerate for $\phi$ $=\pi / 2$ (thicker lines correspond to $\varepsilon_{n}^{+}$). For $\phi=\pi$ all modes are nearly degenerate except the lowest one $\varepsilon_{0}^{-}$.

\section{Electron-hole modes}

For $|q|<|\varepsilon-\mu|$ (setting again $\hbar v \equiv 1$ ) we define the transverse momentum $p$ by the relation

$$
\theta_{+}-\theta_{-}=2 p / E_{T}
$$

The solution to this equation is given by

$$
\varepsilon=p \sqrt{1-\frac{q^{2}}{\mu^{2}-p^{2}}} .
$$

The condition $|q|<|\varepsilon-\mu|$ is equivalent to $|q|<\left|\mu-p^{2} / \mu\right|$ $\equiv q_{c}$. The momentum $q_{c}$ is the location of the cusp in the dispersion relation, beyond which the hole component of the mode vanishes.

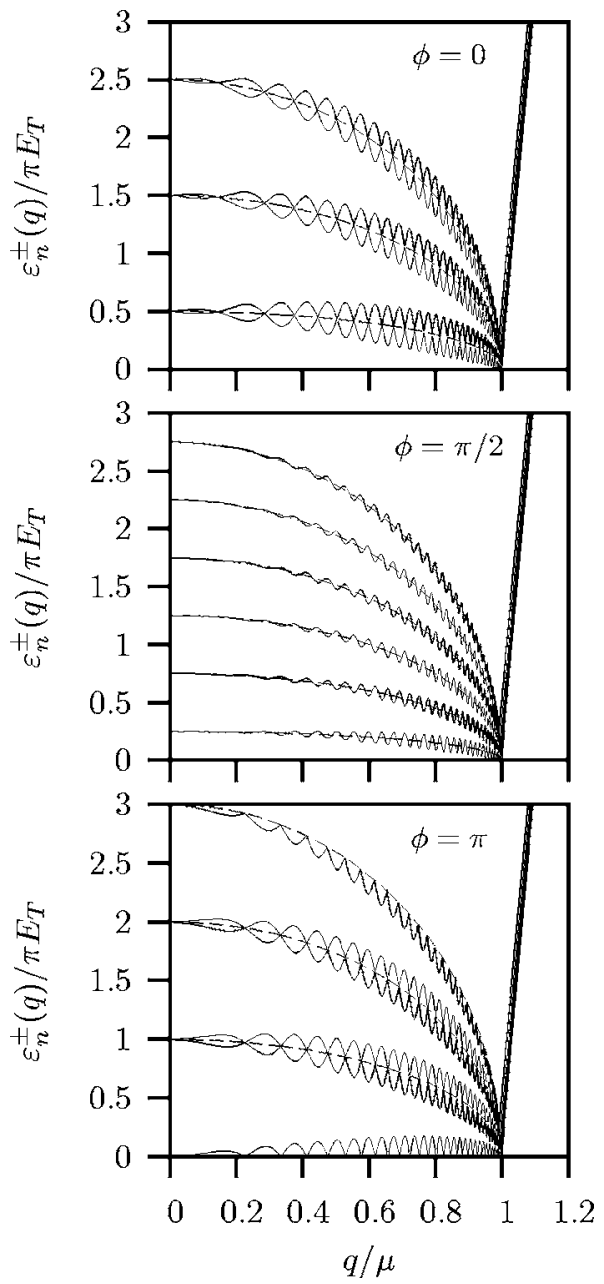

FIG. 3. Same as in Fig. 2 for $\mu / E_{T}=100$. The smoothed dispersion relation (14) is indicated by dashed lines.

If we express $\varepsilon$ in terms of $p$ with the help of Eq. (10), we can write

$$
r=\frac{\left(\mu^{2}-p^{2}\right)^{2}+(\mu q)^{2}}{\left(\mu^{2}-p^{2}\right)^{2}-(\mu q)^{2}}
$$

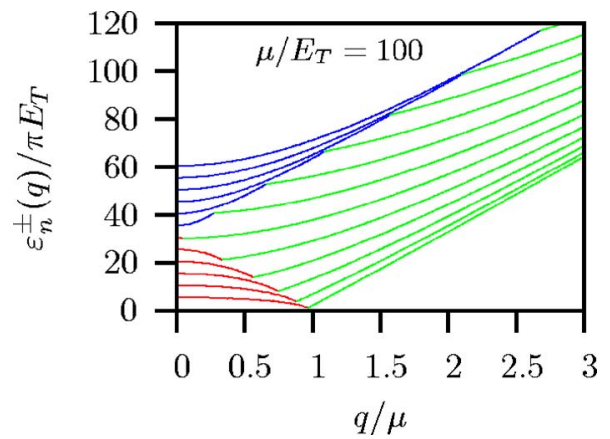

FIG. 4. (Color online) Dispersion relation of the SNS junction, calculated numerically from Eq. (7) for $\phi=0$ and $\mu / E_{T}=100$. The curves show $\varepsilon_{n}^{+}(q)$ with $n=5,10,15, \ldots 60$. The three types of states from Fig. 1 are color coded; red=type $a$, blue=type $b$, green $=$ type $c$. 


$$
\theta_{+}+\theta_{-}=\frac{2 \mu}{E_{T}} \sqrt{1-\frac{q^{2}}{\mu^{2}-p^{2}}} .
$$

This allows us to recast the quantization condition (7) as

$$
\begin{aligned}
& \cos \phi+\cos \left(\frac{2 \mu}{E_{T}} \sqrt{1-\frac{q^{2}}{\mu^{2}-p^{2}}}\right) \\
&=\left(\frac{\mu^{2}-p^{2}}{\mu q}\right)^{2}\left[\cos \phi+\cos \left(2 p / E_{T}\right)\right],
\end{aligned}
$$

which defines the quantization of the transverse momentum $p=p_{n}^{ \pm}$.

For $|q| \ll q_{c}$, the solution to Eq. (12) is given by

$$
p_{n}^{ \pm}=\pi E_{T}\left(n+\frac{1}{2} \pm \frac{\phi}{2 \pi}\right)
$$

with $n=0,1,2, \ldots$ and $\phi \in(-\pi, \pi)$. As $q$ approaches the cusp at $q_{c}$, the first term in Eq. (12) causes the dispersion relation $\varepsilon(q)$ to oscillate rapidly around a smooth curve $\bar{\varepsilon}(q)$. This smoothed dispersion relation is obtained by substitution of Eq. (13) into Eq. (10), resulting in

$$
\begin{aligned}
& \bar{\varepsilon}_{n}^{ \pm}= \pi E_{T}\left(n+\frac{1}{2} \pm \frac{\phi}{2 \pi}\right) \\
& \times \sqrt{1-\frac{q^{2}}{\mu^{2}-\left(\pi E_{T}\right)^{2}\left(n+\frac{1}{2} \pm \phi / 2 \pi\right)^{2}}}, \\
&|q|<q_{c}=\left|\mu-\frac{\left(\pi E_{T}\right)^{2}}{\mu}\left(n+\frac{1}{2} \pm \frac{\phi}{2 \pi}\right)^{2}\right| .
\end{aligned}
$$

The smoothed dispersion for the lowest modes is indicated in Fig. 3 by a dashed line.

To determine also the rapid oscillations, we proceed as follows. The quantization condition for $p$ can be written as

$$
p_{n}^{ \pm}=\pi E_{T}\left(n+\frac{1}{2} \pm \frac{\gamma_{n}^{ \pm}}{2 \pi}\right)
$$

where the phases $\gamma_{n}^{ \pm}$can be determined by iteration from Eq. (12). The first iteration turns out to be already highly accurate in the high-doping regime $\mu \gg E_{T}$. It is given by

$$
\begin{aligned}
\gamma_{n}^{ \pm}= & \arccos \left[\left(1-\frac{(\mu q)^{2}}{\left(\mu^{2}-p^{2}\right)^{2}}\right) \cos \phi\right. \\
& \left.-\frac{(\mu q)^{2}}{\left(\mu^{2}-p^{2}\right)^{2}} \cos \left(\frac{2 \mu}{E_{T}} \sqrt{1-\frac{q^{2}}{\mu^{2}-p^{2}}}\right)\right],
\end{aligned}
$$

where the momentum $p$ on the right-hand side is taken in the zeroth approximation (13). The difference between the approximate analytical results of Eqs. (10), (15), and (16) and the exact numerical results plotted in Fig. 3 are not visible on the scale of that figure.

\section{Electron modes}

For $|q|>|\varepsilon-\mu|$, the angle $\theta_{-}$becomes strictly imaginary. In this interval we define the transverse momentum $p$ by

$$
\theta_{+}=2 p / E_{T} .
$$

The condition $|q|>|\varepsilon-\mu|$ is then still equivalent to $|q|>\mid \mu$ $-p^{2} / \mu \mid \equiv q_{c}$. From Eq. (17) we cast the branch $|q|>q_{c}$ of the dispersion relation in the form

$$
\varepsilon=\sqrt{q^{2}+4 p^{2}}-\mu,
$$

where the momentum $p=p_{n}^{ \pm}$is quantized. The exact quantization condition follows directly from Eq. (7).

For large longitudinal momenta $|q| \gg \max \left(E_{T}, q_{c}\right)$ the reflection angle $\theta_{-} \approx \pm i q / E_{T}$ of the hole takes on large imaginary values. Therefore both $\sin \theta_{-}$and $\cos \theta_{-}$in the quantization condition (7) are exponentially large and the $\phi$ dependence of the solution can be neglected. This shows that the electron modes are insensitive to the superconducting phase difference across the channel.

At the cusp $|q|=q_{c}$ of the disperrsion relation we find $\theta_{-}$ $=0$ and $\varepsilon=p^{2} / \mu$. The coefficient $r$ in Eq. (7) tends to infinity, leading to

$$
\lim _{|q| \rightarrow q_{c}} r \sin \theta_{-}=\frac{\mu^{2}-p^{2}}{p E_{T}} .
$$

The quantization condition at the cusp thus simplifies to

$$
\cos \phi+\cos \left(2 p / E_{T}\right)=\frac{\mu}{E_{T}}\left(\frac{p}{\mu}-\frac{\mu}{p}\right) \sin \left(2 p / E_{T}\right) .
$$

For $\mu \gg E_{T}$ the condition is reduced to $\sin \left(2 p / E_{T}\right)=0$ with the solution

$$
p_{n}^{+}=\pi E_{T}(n+1), \quad p_{n}^{-}=\pi E_{T}\left(n+\frac{1}{2}\right),
$$

which is, again, $\phi$ independent. The quantization condition (21) formally corresponds to $\gamma_{n}^{+}=\pi, \gamma_{n}^{-}=0$ in Eq. (15).

\section{EXCITATION GAP}

At small doping $\mu \lesssim E_{T}$ the excitation gap is given by

$$
E_{\text {gap }}=\frac{1}{2}(\pi-|\phi|) E_{T} \equiv E_{0}(\phi),
$$

which is the energy of the lowest mode at $q=0$. For $\mu \geq E_{T}$ the gap is given by the energy of the lowest mode at a nonzero longitudinal momentum $|q| \lesssim q_{c}$, which corresponds to the deepest minimum of the oscillatory dispersion relation. We have not succeeded in determining this minimum analytically from the quantization condition (7), but we have a very accurate numerical solution. 


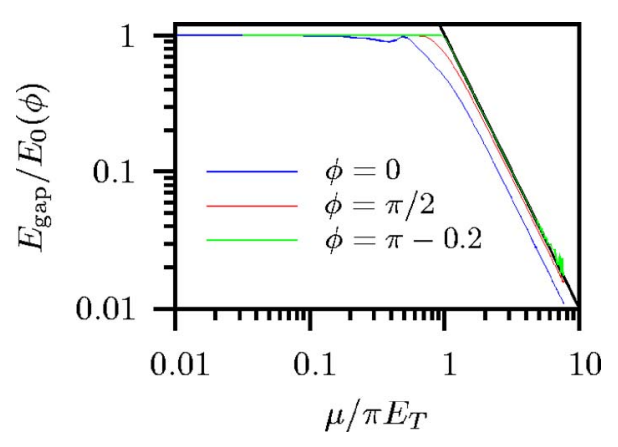

FIG. 5. (Color online) Double-logarithmic plot of the energy dependence of the excitation gap, calculated numerically from Eq. (7), for three different values of the superconducting phase difference $\phi$ (colored lines). The straight black line is the asymptote $E_{\text {gap }} / E_{0}(\phi) \propto\left(\pi E_{T} / \mu\right)^{2}$.

Results for different values of $\phi$ are presented in Fig. 5. One can see that the ratio $E_{\text {gap }} / E_{0}(\phi)$ depends only weakly on the superconducting phase difference $\phi$ and that the crossover to a decay $\propto \mu^{-2}$ happens in a narrow interval around $\mu=\pi E_{T}$. As shown by the black line in Fig. 5, the large- $\mu$ asymptote is given by

$$
E_{\mathrm{gap}}=c(\phi) E_{0}(\phi)\left(\pi E_{T} / \mu\right)^{2},
$$

with $c(\phi)$ increasing from $1 / 2$ at $\phi=0$ to 1 at $|\phi|=\pi$.

\section{DENSITY OF STATES}

\section{A. Thermodynamic limit}

Before turning to the calculation of the density of states at finite $E_{T}$, it is instructive to first determine the behavior in the thermodynamic limit $d \rightarrow \infty \Leftrightarrow E_{T} \rightarrow 0$. The DBdG equation then decouples into separate Dirac equations for electrons and holes. The total density of states $\rho(\varepsilon)=\rho_{e}(\varepsilon)+\rho_{h}(\varepsilon)$ is the sum of the electron density of states $\rho_{e}(\varepsilon) \propto \mu+\varepsilon$ and the hole density of states $\rho_{h}(\varepsilon) \propto|\mu-\varepsilon|$, leading to

$$
\rho(\varepsilon)=\frac{4 \mathcal{L} d}{\pi(\hbar v)^{2}} \max (\mu, \varepsilon), \quad \text { if } \varepsilon \gg E_{T} .
$$

Here $\mathcal{L}$ is the extension of the junction in the $y$ direction and the factor of 4 accounts for the spin and valley degeneracies. [For a derivation of Eq. (24) directly from the quantization condition (7), see the Appendix.]

In Fig. 6 we plot the density of states $\rho$ of the DBdG equation together with the separate electron and hole contributions $\rho_{e}$ and $\rho_{h}$. The superconducting proximity effect will introduce fine structure in $\rho$ on the scale of the Thouless energy $E_{T}$, as we will determine in the next subsections. We consider separately the low-doping regime $\mu \ll E_{T}$, where the contribution from interband electron-hole modes dominates, and the high-doping regime $\mu \gg E_{T}$, where the intraband electron-hole modes dominate.

\section{B. Low-doping regime}

To determine the excitation spectrum in the low-doping regime, we take the $\mu \rightarrow 0$ limit of Eq. (14), resulting in

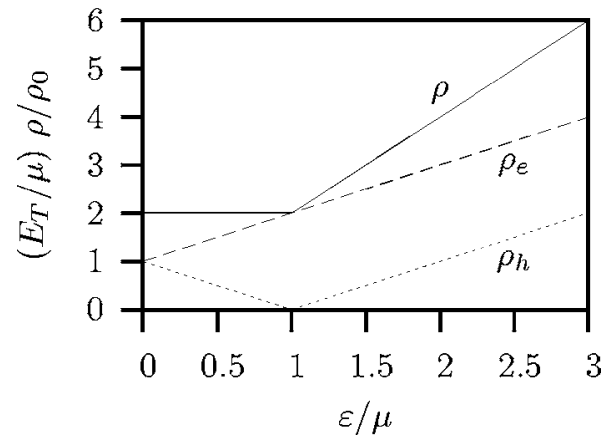

FIG. 6. Thermodynamic limit $E_{T}=\hbar v / d \rightarrow 0$ of the density of states $\rho$ of the SNS junction, according to Eq. (24) with $\rho_{0}$ $=4 \mathcal{L} / \pi \hbar v$. The total density of states $\rho$ (solid) is the sum of the density $\rho_{e} \propto \mu+\varepsilon$ of electron states (dashed) and the density $\rho_{h}$ $\propto|\mu-\varepsilon|$ of hole states (dotted).

$$
\varepsilon_{n}^{ \pm}=\sqrt{(\hbar v q)^{2}+\left(\pi E_{T}\right)^{2}\left(n+\frac{1}{2} \pm \phi / 2 \pi\right)^{2}}
$$

for $n=0,1,2, \ldots$ and $\phi \in(-\pi, \pi)$. (There is no need to distinguish $\bar{\varepsilon}$ from $\varepsilon$, because the dispersion relation does not oscillate in this regime.) The two series of modes $\varepsilon_{n}^{+}$and $\varepsilon_{n}^{-}$ are nondegenerate, except for $\phi=0, \pi$. (The lowest mode $\varepsilon_{0}^{-}$ is nondegenerate also for $\phi=\pi$.)

In Fig. 7 we plot the density of states

$$
\begin{aligned}
\rho(\varepsilon) & =\frac{4 \mathcal{L}}{\pi} \sum_{n=0}^{\infty} \sum_{ \pm}\left|\frac{\partial \varepsilon_{n}^{ \pm}}{\partial q}\right|^{-1}, \\
& =\frac{4 \mathcal{L} \varepsilon}{\pi \hbar v E_{T}} \sum_{n=0}^{\infty} \sum_{ \pm}\left(X_{n}^{ \pm}\right)^{-1 / 2} \Theta\left(X_{n}^{ \pm}\right), \\
X_{n}^{ \pm} & =\left(\varepsilon / E_{T}\right)^{2}-\pi^{2}\left(n+\frac{1}{2} \pm \phi / 2 \pi\right)^{2},
\end{aligned}
$$

with $\Theta$ the unit step function. The excitation spectrum has a gap at the energy $E_{0}$ given by Eq. (2). The gap closes for $|\phi|=\pi$, when $\rho=4 \mathcal{L} / \pi \hbar v \equiv \rho_{0}$ is constant at low energies. At

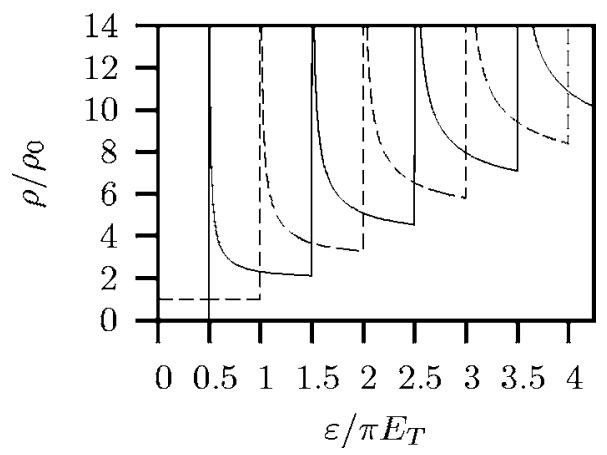

FIG. 7. Density of states of the SNS junction in the low-doping regime, for superconducting phase difference $\phi=0$ (solid curves) and $\phi=\pi$ (dashed curves). The curves are calculated from Eq. (26), normalized by $\rho_{0}=4 \mathcal{L} / \pi \hbar v$. The excitation gap for $\phi=0$ is at $E_{0}$ $=\pi E_{T} / 2$. 
large excitation energies $\varepsilon \gg E_{T}$, the sum over $n$ in Eq. (26) may be replaced by an integral, resulting in a linearly increasing density of states,

$$
\rho(\varepsilon)=\frac{4 \mathcal{L} \varepsilon}{\pi \hbar v E_{T}}, \quad \text { if } \varepsilon \gg E_{T},
$$

in agreement with the thermodynamic limit (24).

The group velocity $v_{n}^{ \pm}$in the $y$ direction of the $n$th mode is given by the derivative

$$
v_{n}^{ \pm}=\frac{\partial \varepsilon_{n}^{ \pm}}{\hbar \partial q}
$$

For each propagating mode $v_{n}^{ \pm} \rightarrow v$ with increasing excitation energy. These are all interband electron-hole modes. The purely electronic modes are pushed to $|q| \gtrsim E_{T}^{2} / \mu \rightarrow \infty$ in the low-doping regime $\mu / E_{T} \rightarrow 0$, while the intraband electronhole modes cannot propagate if $\varepsilon>\mu$.

\section{High-doping regime}

As shown in Sec. II C, the electron-hole branch of the dispersion relation for $\mu \gg E_{T}$ is a rapidly oscillating function. Small local variations in the separation $d$ of the NS interfaces, on the scale of the Fermi wave length $\lambda_{F}=h v / \mu$, will average out these oscillations, leaving the smoothed dispersion relation (14). In the large- $\mu$ limit this reduces to

$$
\bar{\varepsilon}_{n}^{ \pm}=\frac{\pi E_{T}}{\mu}\left(n+\frac{1}{2} \pm \frac{\phi}{2 \pi}\right) \sqrt{\mu^{2}-(\hbar v q)^{2}} .
$$

The branch of purely electronic states (for $\hbar v|q|>\mu$ ) is not described by Eq. (30), but since it contributes negligibly to the density of states for $\varepsilon \ll \mu$ we need not consider it here.

The smoothed density of states is given by

$$
\begin{aligned}
\bar{\rho}(\varepsilon)= & \frac{4 \mathcal{L}}{\pi} \sum_{n=0}^{\infty} \sum_{ \pm}\left|\frac{\partial \bar{\varepsilon}_{n}^{ \pm}}{\partial q}\right|-1 \\
= & \frac{4 \mathcal{L} \mu \varepsilon}{\pi^{2} \hbar v E_{T n=0}^{2}} \sum_{ \pm}^{\infty}\left(Y_{n}^{ \pm}\right)^{-1 / 2} \Theta\left(Y_{n}^{ \pm}\right) \\
& \times\left(n+\frac{1}{2} \pm \phi / 2 \pi\right)^{-1}, \\
Y_{n}^{ \pm}= & \pi^{2}\left(n+\frac{1}{2} \pm \phi / 2 \pi\right)^{2}-\left(\varepsilon / E_{T}\right)^{2} .
\end{aligned}
$$

We plot it in Fig. 8 for $\phi=0$.

The peaks in the density of states at $\varepsilon_{n}^{ \pm}=\pi E_{T}(n$ $\left.+\frac{1}{2} \pm \phi / 2 \pi\right)$ are analogous to the De Gennes-Saint James resonances ${ }^{13}$ in conventional Josephson junctions. The lowest resonance is at the same energy $E_{0}=\frac{1}{2}(\pi-|\phi|) E_{T}$ as the gap (2) in the low-doping regime-however, in the highdoping regime the density of states is gapless, vanishing linearly at small excitation energies with a $\phi$-dependent slope:

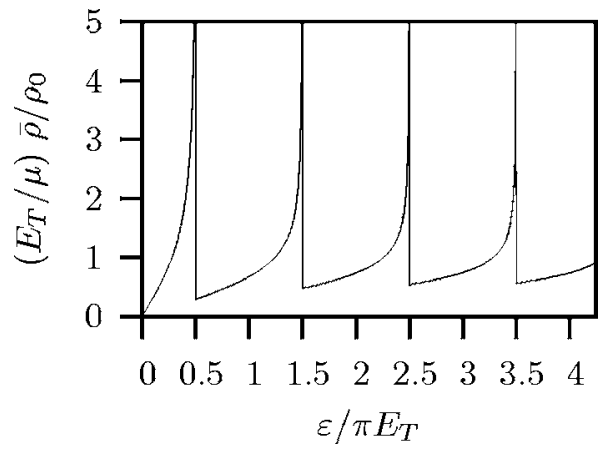

FIG. 8. Smoothed density of states of the SNS junction in the high-doping regime for $\phi=0$, calculated from Eq. (31).

$$
\bar{\rho}(\varepsilon)=\frac{4 \mathcal{L} \mu \varepsilon}{\pi \hbar v E_{T}^{2}} \frac{1}{\cos ^{2}(\phi / 2)}, \quad \text { if } \varepsilon \ll E_{0} .
$$

The slope diverges when $\phi \rightarrow \pi$, because then the lowest resonance is at $\varepsilon=0$. At high excitation energies $\varepsilon \gg E_{T}$ (but still $\varepsilon \ll \mu$ ), the density of states approaches a $\phi$-independent limit,

$$
\bar{\rho}(\varepsilon)=\frac{4 \mathcal{L} \mu}{\pi \hbar v E_{T}}, \quad \text { if } \varepsilon \gg E_{T},
$$

in agreement with Eq. (24).

The group velocity $\bar{v}_{n}^{ \pm}=\partial \bar{\varepsilon}_{n}^{ \pm} / \hbar \partial q$ corresponding to the smoothed density of states is of order $v E_{T} / \mu \simeq v \lambda_{F} / d$ for $\hbar v|q| \ll \mu$, much smaller than the carrier velocity $v$. This is as expected from the classical trajectories in Fig. 1(a).

\section{THERMAL CONDUCTANCE}

The thermal conductance $G_{\text {thermal }}=I_{Q} / \Delta T$ of the graphene channel, for heat flow $I_{Q}$ parallel to the NS boundaries, can be measured by applying a temperature difference $\Delta T=T_{L}$ $-T_{R}$ between the two ends of the channel (see Fig. 9). Experiments of this type have been performed in metals by Eom, Chien, and Chandrasekhar ${ }^{14}$ and analyzed theoretically in Refs. 15 and 16. Because of the large mismatch in Fermi wave vector, the normal region constitutes a "weak link" between the two superconducting regions, which can be maintained at a definite phase difference. ${ }^{17}$ The thermal conductance of mesoscopic SNS junctions was studied recently in Refs. 11 and 18.

To determine the thermal conductance of the graphene channel we start from the Landauer-type formula ${ }^{19-21}$

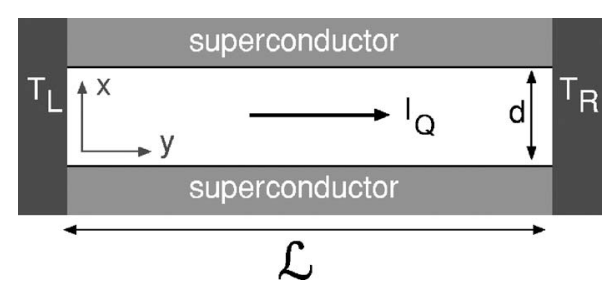

FIG. 9. A temperature difference $\Delta T=T_{L}-T_{R}$ between the two ends of the graphene channel drives a heat current $I_{Q}$, carried by Andreev modes in the normal region at temperatures below the gap $\Delta_{0}$ in the superconductors. 


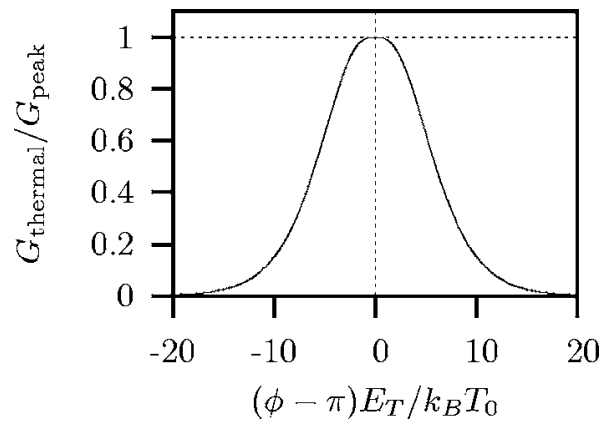

FIG. 10. Thermal conductance of the SNS junction in the lowdoping, low-temperature regime $\left(\mu, k_{B} T_{0} \ll E_{T}\right)$, calculated as a function of the superconducting phase difference $\phi$ (modulo $2 \pi$ ) from Eq. (37). The peak value equals $G_{\text {peak }}=\pi k_{B}^{2} T_{0} / 3 \hbar$, which is half of the thermal conductance quantum (Refs. 22-24) per spin and valley degree of freedom.

$$
G_{\text {thermal }}=-\frac{4}{2 \pi \hbar T_{0}} \int_{0}^{\infty} d \varepsilon \varepsilon^{2} \frac{\partial f}{\partial \varepsilon} \sum_{n} \mathcal{T}_{n}(\varepsilon),
$$

valid for small temperature differences $\Delta T \ll T_{L}, T_{R}$. (The factor of 4 is again from the spin and valley degeneracy.) We assume that the mean temperature $T_{0}=\left(T_{L}+T_{R}\right) / 2$ is much less than $\Delta_{0} / k_{B}$, so that the thermal current through the superconductors is suppressed exponentially. ${ }^{4}$ The function $f(\varepsilon)=\left[1+\exp \left(\varepsilon / k_{B} T_{0}\right)\right]^{-1}$ is the Fermi function and $\mathcal{T}_{n}$ is the transmission probability of the $n$th propagating mode along the channel. In a ballistic channel each of the $N(\varepsilon)$ propagating modes at energy $\varepsilon$ has transmission probability $\mathcal{T}_{n}=1$, so we obtain the thermal conductance

$$
G_{\text {thermal }}=\frac{1}{2 \pi \hbar k_{B} T_{0}^{2}} \int_{0}^{\infty} d \varepsilon \frac{\varepsilon^{2} N(\varepsilon)}{\cosh ^{2}\left(\varepsilon / 2 k_{B} T_{0}\right)} .
$$

In the low-doping, low-temperature limit $\mu, k_{B} T_{0} \ll E_{T}$ only the lowest mode contributes and the thermal conductance reduces to

$$
G_{\text {thermal }}=\frac{k_{B}^{2} T_{0}}{2 \pi \hbar} \int_{E_{0} / k_{B} T_{0}}^{\infty} d x \frac{x^{2}}{\cosh ^{2}(x / 2)},
$$

with $E_{0}$ the gap given by Eq. (2). As illustrated in Fig. 10, the thermal conductance in this low-doping, low-temperature regime vanishes unless the superconducting phase difference $\phi$ is in a narrow interval of order $k_{B} T_{0} / E_{T}$ around $\pi$ (modulo $2 \pi)$. The peak at $\phi=\pi$ has height

$$
G_{\text {peak }}=\frac{\pi}{3} \frac{k_{B}^{2} T_{0}}{\hbar} .
$$

The value $G_{\text {peak }}$ corresponds to a thermal conductance of one-half the quantum value ${ }^{22-24}$

$$
G_{\text {quantum }}=\frac{\pi}{6} \frac{k_{B}^{2} T_{0}}{\hbar},
$$

per spin and valley degree of freedom. The reason for the factor-of-two difference is that, in a normal conductor, every transport channel at the Fermi level can be excited indepen- dently either with electron excitations (filled states just above the Fermi level), or with hole excitations (empty states just below the Fermi level). Each type of excitation contributes an amount $G_{\text {quantum }} / 2$ to $G_{\text {thermal }}$, so that the total thermal conductance per channel is $G_{\text {quantum. }}$. In the SNS junction the superconducting boundaries couple the electron and hole excitations into a single excitation, so that there is only a single contribution of $G_{\text {quantum }} / 2$ per channel to the thermal conductance.

In the high-doping limit $\mu \gg E_{T}$ we may distinguish a moderately low temperature regime $E_{T}^{2} / \mu \ll k_{B} T_{0} \lesssim E_{T}$ and an ultralow temperature regime $k_{B} T_{0} \ll E_{T}^{2} / \mu$. In the ultralow temperature regime it is again only the lowest mode which contributes, so Eq. (37) remains valid if we replace $E_{0}$ by $E_{\text {gap }}$ from Sec. III. In the moderately low temperature regime there remains a large number

$$
N(\varepsilon)=\frac{4}{\pi E_{T}} \sqrt{\varepsilon \mu}+\mathcal{O}(1)
$$

of modes that contributes at energies $\varepsilon \leqq k_{B} T_{0}$. Substitution into Eq. (36) gives the thermal conductance

$$
G_{\text {thermal }}=2.34 \frac{k_{B}^{2} T_{0}}{\hbar} \sqrt{\frac{\mu k_{B} T_{0}}{E_{T}^{2}}} .
$$

The thermal conductance is insensitive to the superconducting phase difference because of the vanishing excitation gap in the high-doping regime.

\section{CONCLUSION}

We have shown that a graphene channel with superconducting boundaries supports a type of propagating modes along the channel that do not exist in usual SNS junctions. These "Andreev modes" exist because the Andreev reflection close to the Dirac point of vanishing Fermi energy $\mu$ is specular. ${ }^{7}$ The Andreev modes are charge neutral, so they transport energy but no charge along the channel.

The thermal conductance due to the Andreev modes depends strongly on the superconducting phase difference $\phi$, because of the $\phi$-dependent excitation gap $E_{0}$ of the Andreev modes. Away from the Dirac point the character of the Andreev reflection changes from specular reflection to retroreflection. The excitation gap closes and the thermal conductance becomes $\phi$ independent.

The closing of the excitation gap with increasing doping can be studied directly by point contact spectroscopy (tunneling into the graphene layer via a tunnel probe on top of the layer).

\section{ACKNOWLEDGMENTS}

This research was supported by the Dutch Science Foundation NWO/FOM and by the German Science Foundation DFG through SFB 513. M.T. acknowledges the hospitality of the Max-Planck-Institute for Physics of Complex Systems in Dresden. 


\section{APPENDIX: DERIVATION OF EQ. (24) FROM THE QUANTIZATION CONDITION}

It is instructive to calculate the density of states in the thermodynamic limit directly from the quantization condition (7). The dispersion relation for all $q$ can be compactly written as

$$
\varepsilon_{n}^{ \pm}(q)= \begin{cases}p \sqrt{1-\frac{q^{2}}{\mu^{2}-p^{2}}}, & q \leqslant\left|\mu-p^{2} / \mu\right|, \\ \sqrt{4 p^{2}+q^{2}}-\mu, & q \geqslant\left|\mu-p^{2} / \mu\right|,\end{cases}
$$

where the momentum $p=p_{n}^{ \pm}$is quantized according to Eqs. (15) and (21). The density of states is given by

$$
\rho(\varepsilon)=\frac{4 \mathcal{L}}{\pi} \sum_{n=0}^{\infty} \sum_{ \pm} \int_{0}^{\infty} d q \delta\left[\varepsilon-\varepsilon_{n}^{ \pm}(q)\right] .
$$

Since the quantization condition of the momentum $p$ is linear in the mode index $n$, we can replace the summation over $n$ with the integration over $p$ in the thermodynamic limit $d \rightarrow \infty$. In this limit we can ignore the dependence of the phases $\gamma_{n}^{ \pm}$on $q$. The integral Eq. (A2) results in

$$
\begin{gathered}
\rho(\varepsilon)=\frac{8 \mathcal{L} d}{(\pi \hbar v)^{2}} \int_{\min \{\varepsilon, \sqrt{\varepsilon \mu}\}}^{\max \{\varepsilon, \sqrt{\varepsilon \mu}\}} d p \frac{\varepsilon}{p} \sqrt{\frac{\mu^{2}-p^{2}}{p^{2}-\varepsilon^{2}}} \\
+\frac{8 \mathcal{L} d}{(\pi \hbar v)^{2}} \int_{0}^{\sqrt{\varepsilon \mu}} d p \frac{\varepsilon+\mu}{\sqrt{(\varepsilon+\mu)^{2}-4 p^{2}}} \\
=\frac{4 \mathcal{L} d}{\pi(\hbar v)^{2}} \max (\varepsilon, \mu) .
\end{gathered}
$$

The first and second integral on the right-hand side of Eq. (A3) are, respectively, the contributions from electron hole and electron modes to the density of states in the thermodynamic limit. Even though each integral is a nontrivial function of energy, their sum reduces to the elementary result (24), confirming the consistency of our analysis.
${ }^{1}$ R. Saito, G. Dresselhaus, and M. S. Dresselhaus, Physical Properties of Carbon Nanotubes (Imperial College, London, 1998).

${ }^{2}$ N. M. R. Peres, A. H. Castro Neto, and F. Guinea, Phys. Rev. B 73, 195411 (2006).

${ }^{3}$ L. Brey and H. A. Fertig, Phys. Rev. B 73, 235411 (2006).

${ }^{4}$ A. F. Andreev, Zh. Eksp. Teor. Fiz. 46, 1823 (1964) [Sov. Phys. JETP 19, 1228 (1964)].

${ }^{5}$ I. O. Kulik, Zh. Eksp. Teor. Fiz. 57, 1745 (1969) [Sov. Phys. JETP 30, 944 (1970)].

${ }^{6}$ A. V. Shytov, P. A. Lee, and L. S. Levitov, Phys. Usp. 41, 207 (1998).

${ }^{7}$ C. W. J. Beenakker, Phys. Rev. Lett. 97, 067007 (2006).

${ }^{8}$ S. Bhattacharjee and K. Sengupta, Phys. Rev. Lett. 97, 217001 (2006).

${ }^{9}$ M. Titov and C. W. J. Beenakker, Phys. Rev. B 74, 041401(R) (2006).

${ }^{10}$ J. C. Cuevas and A. Levy Yeyati, cond-mat/0609138 (unpublished).

${ }^{11}$ N. B. Kopnin, A. S. Mel'nikov, V. I. Pozdnyakova, D. A. Ryzhov, I. A. Shereshevskii, and V. M. Vinokur, Phys. Rev. Lett. 95, 197002 (2005).
${ }^{12}$ Yu. K. Dzhikaev, Zh. Eksp. Teor. Fiz. 68, 295 (1975) [Sov. Phys. JETP 41, 144 (1975)].

${ }^{13}$ P. G. de Gennes and D. Saint-James, Phys. Lett. 4, 151 (1963).

${ }^{14}$ J. Eom, C.-J. Chien, and V. Chandrasekhar, Phys. Rev. Lett. 81, 437 (1998).

${ }^{15}$ E. V. Bezuglyi and V. Vinokur, Phys. Rev. Lett. 91, 137002 (2003).

${ }^{16}$ P. Virtanen and T. T. Heikkilä, Phys. Rev. Lett. 92, 177004 (2004).

${ }^{17}$ K. K. Likharev, Rev. Mod. Phys. 51, 101 (1979).

${ }^{18}$ N. B. Kopnin, A. S. Mel'nikov, and V. M. Vinokur, Phys. Rev. B 70, 075310 (2004).

${ }^{19}$ U. Sivan and Y. Imry, Phys. Rev. B 33, 551 (1986).

${ }^{20}$ P. N. Butcher, J. Phys.: Condens. Matter 2, 4869 (1990).

${ }^{21}$ H. van Houten, L. W. Molenkamp, C. W. J. Beenakker, and C. T. Foxon, Semicond. Sci. Technol. 7, B215 (1992).

${ }^{22}$ J. B. Pendry, J. Phys. A 16, 2161 (1983).

${ }^{23}$ A. Greiner, L. Reggiani, T. Kuhn, and L. Varani, Phys. Rev. Lett. 78, 1114 (1997).

${ }^{24}$ L. G. C. Rego and G. Kirczenow, Phys. Rev. Lett. 81, 232 (1998). 